\title{
A VERY LOW LUMINOSITY X-RAY FLASH: XMM-NEWTON OBSERVATIONS OF GRB 031203
}

\author{
D. Watson, ${ }^{1}$ J. Huorth, ${ }^{1}$ A. Levan, ${ }^{2}$ P. Jakobsson, ${ }^{1}$ P. T. O’Brien, ${ }^{2}$ J. P. Osborne, ${ }^{2}$ K. Pedersen, ${ }^{1}$ \\ J. N. Reeves, ${ }^{3,4}$ J. A. Tedds, ${ }^{2}$ S. A. Vaughan, ${ }^{2}$ M. J. Ward, ${ }^{2}$ and R. Willingale ${ }^{2}$ \\ Received 2004 January 12; accepted 2004 March 4; published 2004 March 25
}

\begin{abstract}
GRB 031203 was observed by XMM-Newton twice, first with an observation beginning $6 \mathrm{hr}$ after the burst and again after 3 days. The afterglow had average $0.2-10.0 \mathrm{keV}$ fluxes for the first and second observations of (4.2 \pm $0.1) \times 10^{-13}$ and $(1.8 \pm 0.1) \times 10^{-13} \mathrm{ergs} \mathrm{cm}^{-2} \mathrm{~s}^{-1}$, respectively, decaying very slowly according to a power law with an index of $-0.55 \pm 0.05$. The prompt soft X-ray flux, inferred from a detection of the dust echo of the prompt emission, strongly implies that this burst is very soft and should be classified as an X-ray flash (XRF) and further, implies a steep temporal slope $(\Sigma-1.7)$ between the prompt and afterglow phases or in the early afterglow, very different from the later afterglow decay slope. A power law $(\Gamma=1.90 \pm 0.05)$ with absorption at a level consistent with the Galactic foreground absorption fits the afterglow spectrum well. A bright low-redshift $(z=0.105)$ galaxy lies within 0".5 of the X-ray position and is likely to be the gamma-ray burst (GRB) host. At this redshift, GRB 031203 is the closest GRB or XRF known after GRB 980425. It has a very low equivalent isotropic gamma-ray energy in the burst $\left(\sim 3 \times 10^{49} \mathrm{ergs}\right)$ and X-ray luminosity in the afterglow $\left(9 \times 10^{42} \mathrm{ergs} \mathrm{s}^{-1}\right.$ at $\left.10 \mathrm{hr}\right), 3-4$ orders of magnitude less than typical bursts, though higher than either the faint XRF 020903 or GRB 980425 . The rapid initial decline and subsequent very slow fading of the X-ray afterglow is also similar to that observed in GRB 980425, indicating that GRB 031203 may be representative of low-luminosity bursts.
\end{abstract}

Subject headings: gamma rays: bursts — supernovae: general — X-rays: general

On-line material: color figures

\section{INTRODUCTION}

A class of very soft burst, very similar to gamma-ray bursts (GRBs), has been identified and is referred to as X-ray flashes (XRFs; Heise et al. 2001). Given the similarity of durations among XRFs, X-ray-rich bursts, and GRBs (Heise et al. 2001; Barraud et al. 2003), the continuum of spectral properties observed (Lamb, Donaghy, \& Graziani 2003), from classic GRBs to X-ray-rich bursts to XRFs and their cosmological origin (Bloom et al. 2003b; Soderberg et al. 2004), it seems probable that XRFs and GRBs have very similar origins. XRFs have been defined by a larger X-ray than gamma-ray fluence in the burst $\left(S_{\mathrm{X}} / S_{\gamma}>1\right.$; Lamb et al. 2003); their distinguishing characteristic is a shift in the peak energy $\left(E_{\text {peak }}\right)$ of the burst from GRBs (typically $200 \mathrm{keV}$; Preece et al. 2000) to XRFs ( $\$ 50 \mathrm{keV}$; Preece et al. 2000; Lamb et al. 2003). While GRBs and XRFs are located at cosmological distances, few have been located at redshifts less than 0.3. They are GRB 030329 at $z=$ 0.1685 (associated with SN 2003dh; Hjorth et al. 2003; Stanek et al. 2003), XRF 020903 with its probable host galaxy at $z=0.251$ (Soderberg et al. 2004), and GRB 980425, probably associated with SN 1998bw at $z=0.0085$ (Galama et al. 1998). Of these, only the redshift for GRB 030329 was found directly from the afterglow, and it has characteristics that are still a matter of some debate; XRF 020903 had a very low peak spectral energy and a low luminosity (Sakamoto et al. 2004; Soderberg et al. 2004), and GRB 980425 had an ex-

\footnotetext{
${ }^{1}$ Niels Bohr Institute, Astronomical Observatory, University of Copenhagen, Juliane-Maries Vej 30, DK-2100 Copenhagen Ø, Denmark; darach@ astro.ku.dk, jens@astro.ku.dk, pallja@astro.ku.dk, kp@astro.ku.dk.

${ }^{2}$ X-Ray Astronomy Group, Department of Physics and Astronomy, Leicester University, Leicester LE1 7RH, UK; anl@star.le.ac.uk, pto@star.le.ac.uk, julo@star.le.ac.uk, jat@star.le.ac.uk, sav2@star.le.ac.uk, mjw@star.le.ac.uk, rw@star.le.ac.uk.

${ }^{3}$ Laboratory for High Energy Astrophysics, Code 662, NASA Goddard Space Flight Center, Greenbelt, MD 20771; jnr@milkyway.gsfc.nasa.gov.

${ }^{4}$ Universities Space Research Association.
}

traordinarily low luminosity (Kulkarni et al. 1998). On the basis of GRB 980425, it was suggested that a variety of GRBs with very low luminosity, detectable at low redshift, form a distinct class of objects from classical high-redshift GRBs (Kulkarni et al. 1998). It has also been suggested that low luminosity in a burst is related to a steeply declining afterglow ("f-GRBs"; Bloom, Frail, \& Kulkarni 2003c) or to low $E_{\text {peak }}$ (Amati et al. 2002; Barraud et al. 2003; Sakamoto et al. 2004).

In this Letter, we report on XMM-Newton observations of GRB $031203,{ }^{5}$ begun $6 \mathrm{hr}$ after the burst, as well as a second, serendipitous observation made on 2003 December 6. During the first observation, an echo of the prompt emission, reflected off dust in our Galaxy, was detected as an expanding ring centered on the afterglow. This was reported by Vaughan et al. (2004), and that remarkable detection (the first time that we are aware that prompt X-rays have been detected at energies below $2 \mathrm{keV}$ ) has allowed us to infer properties of the burst itself in soft Xrays; however, the dust echo is not directly considered here.

A cosmology with $\Omega_{m}=0.3, \Omega_{\Lambda}=0.7$, and $H_{0}=75$ $\mathrm{km} \mathrm{s}^{-1} \mathrm{Mpc}^{-1}$ is assumed throughout. Error ranges quoted are $90 \%$ confidence intervals unless stated otherwise.

\section{OBSERVATIONS AND DATA REDUCTION}

An initial $56 \mathrm{ks}$ exposure, centered on the error circle provided by the INTEGRAL burst-alert system (IBAS; Mereghetti et al. 2003; Götz et al. 2003), was made with XMM-Newton's EPIC cameras. One X-ray source close to the center of the circle was observed to fade (Rodriquez-Pascual et al. 2003). The astrometry of the field was corrected by matching X-ray sources to stars from the USNO-A2.0 catalog using the SAS task EPOSCORR (Tedds \& Watson 2004). The position of the fading X-ray source, R.A. $=08^{\mathrm{h}} 02^{\mathrm{m}} 30^{\mathrm{s}} \cdot 190$, decl. $=-39^{\circ} 51^{\prime} 04^{\prime \prime}$.05 (J2000.0), with a

\footnotetext{
${ }^{5}$ We refer to this burst as GRB 031203 to avoid confusion, though we show it to be an XRF in $\S 5$.
} 
$1 \sigma$ error radius of 0.77 (Tedds et al. 2003), is consistent with the position of a fading radio source (Frail 2003; Soderberg, Kulkarni, \& Frail 2003). A star-forming galaxy at redshift $z=$ 0.105 (Hsia et al. 2003; Bloom et al. 2003a; Prochaska et al. 2004) was also detected within 0 ".5 of this position at nearinfrared wavelengths. No other fading sources are detected with EPIC in the IBAS error circle. In light of this and the fact that the dust echo of a very bright transient source is detected centered on the fading source (Vaughan et al. 2004), the X-ray source at this position must be the GRB afterglow.

The first XMM-Newton observation was carried out under low background conditions (the background represents $\sim 10 \%$ of the extracted counts), and the data were not filtered for this reason beyond standard processing. Spectral data from the second observation were filtered because of high background in the middle and end of the observation. The "thin" and "medium" filters were used in the first observation with the pn and MOS cameras, respectively. The "thick" filters were used with each camera during the second observation, in which the principle target was $\zeta$ Puppis, and the afterglow was off-axis by $\sim 8^{\prime}$. Data from the EPIC-MOS and pn cameras are consistent within cross-calibration uncertainties. Because of the extreme lack of counts at low energies, most of the counts at energies below $0.8 \mathrm{keV}$ are redistributed from higher energies; lowlevel uncertainty in this redistribution, usually negligible in spectra with only moderate soft absorption, has caused us to exclude data below $0.8 \mathrm{keV}$ from the spectral analysis. The data reduction followed a standard procedure similar to that outlined in Watson et al. (2002b) except that the data were processed and reduced with the XMM-Newton SAS version 5.4.1. A spectral binning using a minimum of 20 counts bin $^{-1}$ was used. Two regions on the detector were used to obtain different background spectra, avoiding obvious sources and the dust echo. No significant difference was observed using either background region. To calibrate the absolute fluxes for the light curves, the mean flux for the observation, derived from the best-fit model to the joint fit of the EPIC spectra, was used to scale the count-rate light curves, using the response matrices and ancillary response vectors generated by the SAS tasks RMFGEN and ARFGEN, which take into account, among other things, the off-axis angle of the source and the filter used. Careful analysis of the flux calibration required the use of ancillary response vectors newer than those available in the current version of the SAS; though usually insignificant for on-axis sources, this does affect sources far from the optical axis considerably (R. Saxton 2004, private communication). The first observation was divided into five $11 \mathrm{ks}$ segments to examine spectral evolution.

\section{RESULTS}

The afterglow decays (Rodriquez-Pascual et al. 2003) during the first observation (Fig. 1) and is well fitted $\left(\chi^{2}=44.9\right.$ for 54 degrees of freedom) by a power law with index $-0.54 \pm$ 0.09 , a decay slower than reported for any previous X-ray afterglow except GRB 980425 (Pian et al. 2000).

Continuing the power-law decay observed in the first observation fits the second observation well $\left(\chi^{2}=46.6\right.$ for both data sets). The fit to both data sets, a power law with index $-0.55 \pm 0.05$, is not improved significantly by the addition of a break to the single power-law model $\left(\chi_{v}^{2}=45.6 / 56\right.$ compared to $\chi_{\nu}^{2}=46.3 / 58, F$-test probability $\left.=0.65\right)$.

We (Vaughan et al. 2004) have discovered the first dust echo observed from a GRB and, using it, have derived the 0.2-10.0 $\mathrm{keV}$ absorbed flux of the prompt emission [(1.5 \pm 0.8$) \times 10^{-7}$

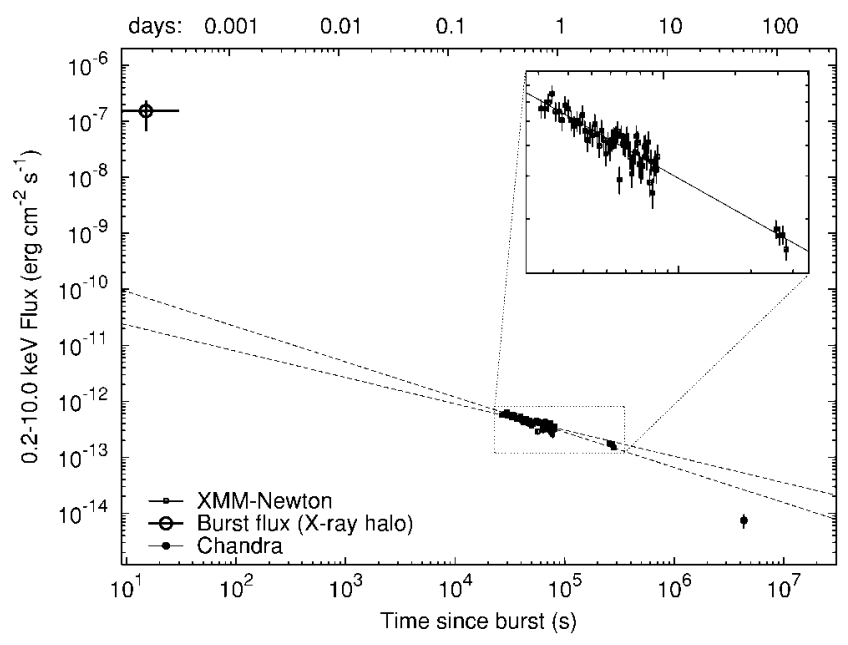

FIG. 1.-X-ray light curve (based on the mean EPIC 0.2-10.0 keV absorbed fluxes) for the afterglow of GRB 031203 fitted with a single power-law model. The $3 \sigma$ limits of the fit are plotted on the main figure (dashed line), while the best fit is shown on the inset. The temporal slope derived from the XMMNewton observations is much too flat $(-0.55 \pm 0.05)$ to fit the prompt flux (open circle) as derived from the observed dust echo. A slope $\lesssim-1.7$ is required to connect the prompt emission with the afterglow flux. The late-time flux detected by Chandra (Fox et al. 2004) is shown for completeness and implies a steepening in the light curve after about 3 days. [See the electronic edition of the Journal for a color version of this figure.]

ergs $\mathrm{cm}^{-2} \mathrm{~s}^{-1}$ ]. The drop in flux to $5 \times 10^{-13}$ ergs $\mathrm{cm}^{-2} \mathrm{~s}^{-1}$ observed in the afterglow at the beginning of the XMM-Newton observation requires a slope $\sim-1.7$ and is not consistent with the decay slope of the best-fitting power law. There must therefore have been a discontinuous flux change of orders of magnitude between the prompt and afterglow phases in the soft X-ray band (a characteristic somewhat atypical of bursts observed by BeppoSAX; Frontera et al. 2000), or there was a faster decay rate in the afterglow phase than is usual in early X-ray afterglows (typical early X-ray afterglow slopes are $\sim-1$ at $t \lesssim 1$ day, e.g., Butler et al. 2003; Tiengo et al. 2003; Watson et al. 2002a, 2003; Mereghetti et al. 2003), which then slowed markedly in the afterglow phase. This light curve is reminiscent of the optical light curves of GRBs 990123 and 021211, where a rapid decline was observed initially ( $\leq 0.1$ days), then a slower decay and finally a resteepening (Fruchter et al. 1999; Castro-Tirado et al. 1999; Li et al. 2003).

The complete spectra of the afterglow from the first and second observations (Fig. 2) and the five $11 \mathrm{ks}$ spectra from the first observation can be fitted with a power law with absorption in excess of the Galactic value $\left(5.9 \times 10^{21} \mathrm{~cm}^{-2}\right.$; Dickey \& Lockman 1990). The best-fit power-law photon indices $\left[N(E) \propto E^{-\Gamma}\right]$ are $1.90 \pm 0.05$ and $1.7 \pm 0.2$ for the first and second observations, respectively, when the absorption was fixed to that found for the combined fit to the dust echo and the afterglow spectra $\left(8.8 \times 10^{21} \mathrm{~cm}^{-2}\right.$; Vaughan et al. 2004). The absorption is consistently $\sim 50 \%$ above the Galactic column density, but this may be due to variations in the local column density on scales $\lesssim 1^{\circ}$ (Dickey \& Lockman 1990). The observed timeaveraged fluxes are $(4.2 \pm 0.1) \times 10^{-13}$ and $(1.8 \pm 0.1) \times$ $10^{-13} \mathrm{ergs} \mathrm{cm}^{-2} \mathrm{~s}^{-1}$.

\section{A LOW-LUMINOSITY BURST AT $z=0.105$}

No optical afterglow was detected for this burst; the upper limit on the flux of the transient based on an extrapolation of the Xray power law at $\sim 0.5$ day $(\Gamma=1.9)$ is $I>21.9$ mag, including 


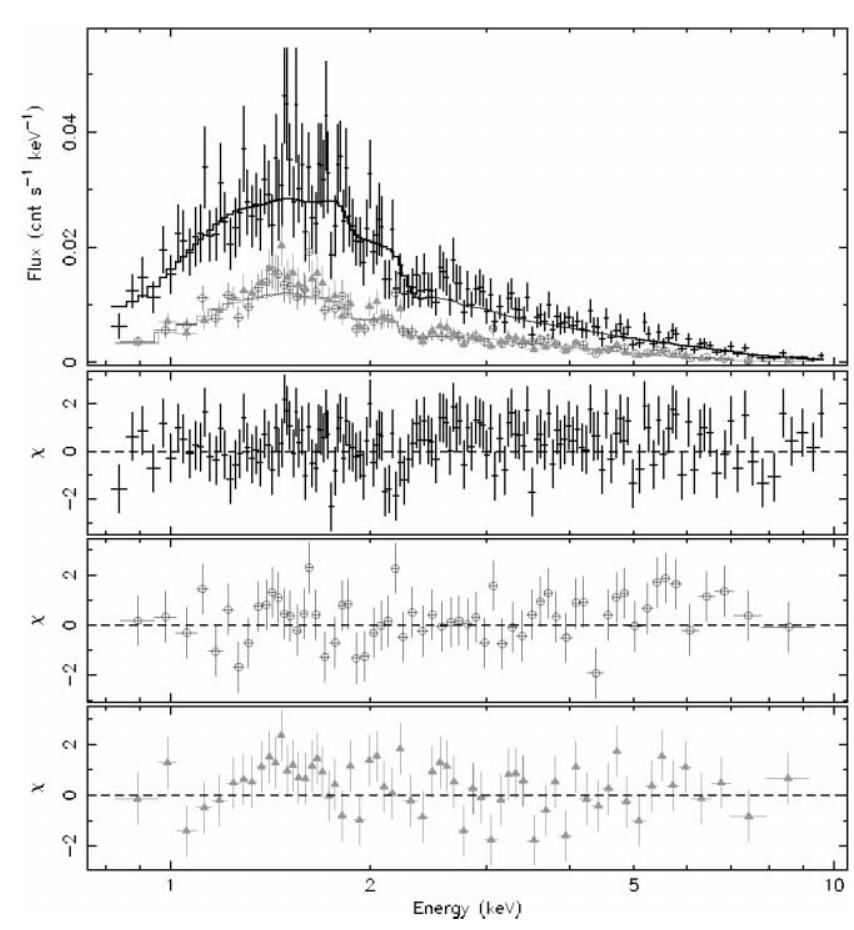

FIG. 2.-EPIC-pn (plus signs), MOS1 (circles), and MOS2 (triangles) spectra of the complete exposure on 2003 December 4 of the afterglow of GRB 031203. The best-fit absorbed power-law model and residuals are also plotted. The effects of the detector response have not been unfolded out of the data. [See the electronic edition of the Journal for a color version of this figure.]

a reddening correction of $A_{I}=2.0$ mag. Given the brightness of the underlying galaxy ( $I=19.1 \mathrm{mag}$; Bailyn et al. 2003), a source with observed $I>21.9$ mag seems unlikely to be detected.

The nondetection of the optical afterglow makes the association of the GRB with the star-forming galaxy at $z=$ 0.105 uncertain since we do not have a redshift associated with the afterglow. The X-ray observations strengthen this association. The X-ray position is the best localization available and places the GRB within $\sim 1 \mathrm{kpc}$ of the center of the galaxy; the probability (based on the I-band galaxy counts of Postman et al. 1998 and neglecting gravitational lensing and extragalactic absorption) of the center of a galaxy at least this bright appearing coincident with the XMM-Newton position by chance is low, $\sim 7 \times 10^{-5}$. The probability of the center of any galaxy being detected (assuming a limiting $I$-band magnitude of 23) within 0.7 radius is only 0.004 .

At a luminosity distance of $453 \mathrm{Mpc}$, GRB 031203 is the closest GRB/XRF with known redshift after GRB 980425. This distance implies that the gamma-ray burst energy and afterglow luminosity were very low compared to cosmological GRBs (Soderberg et al. 2003).

Mereghetti \& Götz (2003) quote a peak 20-200 keV photon flux for GRB 031203 of $1.2 \mathrm{~cm}^{-2} \mathrm{~s}^{-1}$ and a duration of $\sim 30 \mathrm{~s}$. For the following calculations we use an estimate of the mean flux of one-third the peak flux (Hakkila et al. 2000) to derive a photon fluence of $12 \mathrm{~cm}^{-2}$. We also use a power-law index $\Gamma=2.5 \pm 0.3$ for the burst, justified below ( $\$ 5$ ).

The equivalent isotropic gamma-ray energy $(20-2000 \mathrm{keV}$ rest frame) is only $3 \times 10^{49} \mathrm{ergs}$, well below the total gammaray energy in the general case $\left(\sim 10^{51}\right.$ ergs; Bloom et al. 2003c) but a few times greater than XRF 020903 (Sakamoto et al. 2004) and 30 times that of GRB 980425 (Pian et al. 2000). The peak radio luminosity is often used as a surrogate for the total burst energy; GRB 031203's radio luminosity after 4 days (Frail 2003) is very low but within a factor of 3 of GRB 980425/SN 1998bw at the same time (Kulkarni et al. 1998; Soderberg et al. 2004), indicating that it may have an unusually low peak radio luminosity. The X-ray luminosity for GRB 031203 is also low (Soderberg et al. 2003). Even the isotropic equivalent luminosity based on the observed $2-10 \mathrm{keV}$ flux is $9 \times 10^{42} \mathrm{ergs} \mathrm{s}^{-1}$ at $10 \mathrm{hr}$, more than 3 orders of magnitude below the typical value $\left(\sim 5 \times 10^{46} \mathrm{ergs} \mathrm{s}^{-1}\right.$; Berger, Kulkarni, \& Frail 2003), though a factor of $\sim 100$ above GRB 980425 (Pian et al. 2000).

\section{GRB 031203 AS AN XRF}

The photon power-law index from the $0.2-10.0 \mathrm{keV}$ photon fluence (estimated from the dust echo) to the $20-200 \mathrm{keV}$ photon fluence is $\Gamma=2.5 \pm 0.3$. Furthermore, a power-law fit to the dust echo, corrected for the effects of the dust scattering, yields an intrinsic $\Gamma=2.2 \pm 0.3$. A single power law (with $\Gamma \geqslant 2$ ) from 1 to $200 \mathrm{keV}$ is consistent with these numbers: in this case the $E_{\text {peak }}$ of the burst must be below $1 \mathrm{keV}$, a remarkably low $E_{\text {peak }}$ even for an XRF. They are also consistent with a powerlaw index that steepens between the soft $(0.2-10.0 \mathrm{keV})$ and hard (20-200 keV) X-ray bands, with $E_{\text {peak }}$ probably below the 20$200 \mathrm{keV}$ band. It is likely that the $E_{\text {peak }}$ of GRB 031203 was $\ 20$ $\mathrm{keV}$. Dar (2003) predicted $E_{\text {peak }}$ for this burst close to $20 \mathrm{keV}$ based on the cannon ball model; we cannot exclude this possibility. In any case, GRB 031203 is an XRF; the ratio used to define XRFs, $\log \left(S_{\mathrm{X}_{2-30 \mathrm{keV}}} / S_{\gamma_{30-40 \mathrm{k} \mathrm{kev}}}\right)>0$, is $0.6 \pm 0.3$ in this case, an extreme value (compare XRF 020903 with a ratio of 0.7 ; Sakamoto et al. 2004), assuming a single power law in the $2-400 \mathrm{keV}$ band.

We assume above that the fluence observed in the X-ray halo is due entirely to the burst event. Some fraction will, of course, be contributed by the afterglow, since the time of the observed $\mathrm{X}$-ray fluence is only constrained to $\sim 1 \mathrm{hr}$ of the burst. To quantify this, we derive the maximum afterglow contribution to the fluence (where the afterglow flux starts at the mean burst flux and decays without a break to the flux observed at the start of the XMM-Newton observations). This depends on the mean flux of the burst and therefore on the burst duration. The afterglow contributes less than $60 \%$ of the total fluence when the burst duration is $30 \mathrm{~s}$. Even at 60\%, GRB 031203 is still an XRF, with $\log \left(S_{\mathrm{X}} / S_{\gamma}\right) \sim 0.3$ within the uncertainty mentioned above. The contribution of the afterglow to the observed soft Xray fluence cannot influence the classification of GRB 031203 as an XRF. In order for GRB 031203 not to be an XRF, a very large fraction of the fluence would have to be significantly earlier or later than the hard X-ray burst and (somewhat arbitrarily) renamed a "precursor" or a "rebrightening," with a total energy comparable to the burst itself.

INTEGRAL's SPI-ACS detected GRB 031203; the count rate is not inconsistent with that expected from the fluence and spectral slope mentioned above (von Kienlin et al. 2003). If the ratio of peak count rate to mean count rate (Prochaska et al. 2004) in the IBIS band $(20-200 \mathrm{keV})$ is the same as in the SPI-ACS band (>100 keV), then the total $20-200 \mathrm{keV}$ fluence estimated above will be smaller by a factor of 3.2, making GRB 031203 the most extreme XRF ever observed, with $\log \left(S_{\mathrm{X}_{2-30 \mathrm{kev}}} / S_{\gamma_{30-400 \mathrm{kev}}}\right) \sim 1$. An analysis of all the INTEGRAL data will be interesting since the quoted peak flux for the burst $\left(1.3 \times 10^{-7} \mathrm{ergs} \mathrm{cm}^{-2} \mathrm{~s}^{-1}\right.$ in the 20-200 keV band) implies a much harder spectrum during the peak $(\Gamma \sim 1.3)$, indicating strong spectral evolution. This has no impact on our calculations above since they are based on the total fluences in the burst, derived from the count rates only, not the peak flux. A tantalizing result we can compute is an approximate 
upper limit on the pseudo- $z$ ( $\hat{z}$; Atteia 2003) using $E_{\text {peak }}<20 \mathrm{keV}$, $\Gamma>1.9$, and $t_{90}=30 \mathrm{~s}$, for which we find $\hat{z} \lesssim 0.15$.

\section{DISCUSSION}

Amati et al. (2002) have found a relation between the equivalent isotropic gamma-ray total energy $\left(E_{\text {iso }}\right)$ and $E_{\text {peak }}$ in GRBs. Though only a single burst, the low-luminosity XRF 020903 has extended this relation to very low luminosities and peak energies (Sakamoto et al. 2004). Using the best-fit values for this relation quoted by Sakamoto et al. (2004) and the equivalent isotropic luminosity in the $1 \mathrm{keV}-10 \mathrm{MeV}$ band derived here $\left(1.5 \times 10^{50} \mathrm{ergs}\right)$, the $E_{\text {peak }}$ of GRB 031203 should lie close to $\sim 10 \mathrm{keV}$; this is consistent with the $E_{\text {peak }}$ suggested above and further confirms the correlation between peak energies and $E_{\text {iso }}$.

One implication of this correlation is clear; XRFs have low $E_{\text {iso }}$ as we see here. Furthermore, if the total energy in gammarays is nearly constant at $\sim 10^{51}$ ergs (Frail et al. 2001; Bloom et al. 2003c), it implies that the opening angle in XRFs is extremely wide. However, this becomes problematic if there is a significant population of XRFs with peak energies below $\sim 20 \mathrm{keV}$, since the Amati et al. (2002) relation implies that $E_{\text {iso }}$ for these bursts is less than the total energy in gamma rays inferred by Frail et al. (2001). Only one XRF so far had clearly indicated this paradoxXRF 020903, mentioned above. But now GRB 031203 similarly shows a very low $E_{\text {iso }}$. (We note that it has been suggested that XRF 030723 also has a very low $E_{\text {iso }}$ [Lamb et al. 2004], but the redshift in that case is highly uncertain; Fynbo et al. 2004.) These results hint that a significant population of (so far, rare) low- luminosity bursts may indeed exist as required in some models of off-axis GRBs (e.g., Yamazaki et al. 2004).

It has been suggested (Bloom et al. 2003c) that a class of underluminous GRBs exist with fast decay rates (f-GRBs) at early times ( $t \lesssim 0.5$ days). We cannot exclude GRB 031203 from such a class. Both its luminosity and its decay rates are extraordinary; the luminosity is very low and the decay rate unusually slow, but there must be a fast decay in the afterglow before the XMM-Newton observation at less than 0.25 days, if the prompt and afterglow fluxes decay smoothly and there is no abrupt drop in the flux between prompt and afterglow phases. GRB 980425, possibly similar in terms of its low luminosity, is also interesting as a comparison object in terms of its X-ray decay structure. GRB 980425 also showed a fast decay from the prompt X-ray flux (power-law index, $\alpha \sim-1.5$ ) and a very slow decay in X-rays from 1 to 100 days ( $\alpha \sim-0.2)$. In XRF 030723 a very slow decay was observed at optical wavelengths at early times (Fynbo et al. 2004): perhaps very slow early decay rates are related to the apparent luminosity and $E_{\text {peak }}$ of the burst, lending some support to the idea that these bursts may be viewed off-axis (Granot et al. 2002; Yamazaki et al. 2004).

We thank R. Saxton for help with the EPIC flux calibration and the XMM-Newton project scientist and SOC staff for continuing rapid response GRB observations, recognizing the operational load these place on the project. We acknowledge benefits from collaboration within the EU FP5 Research Training Network, "GammaRay Bursts: An Enigma and a Tool." This work was also supported by the Danish Natural Science Research Council (SNF).

\section{REFERENCES}

Amati, L., et al. 2002, A\&A, 390, 81

Atteia, J.-L. 2003, A\&A, 407, L1

Bailyn, C., van Dokkum, P., Buxton, M., Cobb, B., \& Bloom, J. S. 2003, GCN Circ. 2486 (http://gcn.gsfc.nasa.gov/gen/gen3/2486.gen3)

Barraud, C., et al. 2003, A\&A, 400, 1021

Berger, E., Kulkarni, S. R., \& Frail, D. A. 2003, ApJ, 590, 379

Bloom, J. S., Bailyn, C., Buxton, M., Cobb, B., van Dokkum, P., \& Gonzalez, D. 2003a, GCN Circ. 2481 (http://gcn.gsfc.nasa.gov/gen/gen3/2481.gcn3)

Bloom, J. S., Fox, D., van Dokkum, P. G., Kulkarni, S. R., Berger, E., Djorgovski, S. G., \& Frail, D. A. 2003b, ApJ, 599, 957

Bloom, J. S., Frail, D. A., \& Kulkarni, S. R. 2003c, ApJ, 594, 674

Butler, N. R., Marshall, H. L., Ricker, G. R., Vanderspek, R. K., Ford, P. G., Crew, G. B., Lamb, D. Q., \& Jernigan, J. G. 2003, ApJ, 597, 1010

Castro-Tirado, A. J., et al. 1999, Science, 283, 2069

Dar, A. 2003, GCN Circ. 2485 (http://gcn.gsfc.nasa.gov/gcn/gcn3/2485.gcn3)

Dickey, J. M., \& Lockman, F. J. 1990, ARA\&A, 28, 215

Fox, D. B., Soderberg, A. M., Kulkarni, S. R., \& Frail, D. 2004, GCN Circ. 2522 (http://gcn.gsfc.nasa.gov/gcn/gcn3/2522.gcn3)

Frail, D. A. 2003, GCN Circ. 2473 (http://gcn.gsfc.nasa.gov/gen/gcn3/2473.gcn3)

Frail, D. A., et al. 2001, ApJ, 562, L55

Frontera, F., et al. 2000, ApJS, 127, 59

Fruchter, A. S., et al. 1999, ApJ, 519, L13

Fynbo, J. P. U., et al. 2004, ApJ, submitted (astro-ph/0402240)

Galama, T. J., et al. 1998, Nature, 395, 670

Götz, D., Mereghetti, S., Beck, M., Borkowski, J., \& Mowlavi, N. 2003, GCN Circ. 2459 (http://gcn.gsfc.nasa.gov/gcn/gcn3/2459.gcn3)

Granot, J., Panaitescu, A., Kumar, P., \& Woosley, S. E. 2002, ApJ, 570, L61

Hakkila, J., Haglin, D. J., Pendleton, G. N., Mallozzi, R. S., Meegan, C. A., \& Roiger, R. J. 2000, ApJ, 538, 165

Heise, J., in 't Zand, J., Kippen, R. M., \& Woods, P. M. 2001, in GammaRay Bursts in the Afterglow Era, ed. E. Costa, F. Frontera, \& J. Hjorth (Berlin: Springer), 16

Hjorth, J., et al. 2003, Nature, 423, 847

Hsia, C. H., Lin, H. C., Huang, K. Y., Urata, Y., Ip, W. H., \& Tamagawa, T. 2003, GCN Circ. 2470 (http://gcn.gsfc.nasa.gov/gcn/gcn3/2470.gcn3)

Kulkarni, S. R., et al. 1998, Nature, 395, 663

Lamb, D. Q., Donaghy, T. Q., \& Graziani, C. 2003, ApJ, submitted (astro-ph/ 0312634)
Lamb, D. Q., et al. 2004, in Proc. Second VERITAS Symp. on TeV Astrophysics, in press (astro-ph/0310414)

Li, W., Filippenko, A. V., Chornock, R., \& Jha, S. 2003, ApJ, 586, L9

Mereghetti, S., \& Götz, D. 2003, GCN Circ. 2460 (http://gcn.gsfc.nasa.gov/ $\mathrm{gcn} / \mathrm{gcn} 3 / 2460 . \mathrm{gcn} 3)$

Mereghetti, S., et al. 2003, ApJ, 590, L73

Mereghetti, S., Götz, D., Borkowski, J., Walter, R., \& Pedersen, H. 2003, A\&A, 411, L291

Pian, E., et al. 2000, ApJ, 536, 778

Postman, M., Lauer, T. R., Szapudi, I., \& Oegerle, W. 1998, ApJ, 506, 33

Preece, R. D., Briggs, M. S., Mallozzi, R. S., Pendleton, G. N., Paciesas, W. S., \& Band, D. L. 2000, ApJS, 126, 19

Prochaska, J. X., et al. 2004, ApJ, submitted (astro-ph/0402085)

Rodriquez-Pascual, P., Santos-Lléo, M., Gonzalez-Riestra, R., Schartel, N., \& Altieri, B. 2003, GCN Circ. 2474 (http://gcn.gsfc.nasa.gov/gen/gcn3/2474 gcn 3$)$

Sakamoto, T., et al. 2004, ApJ, 602, 875

Soderberg, A. M., Kulkarni, S. R., \& Frail, D. A. 2003, GCN Circ. 2483 (http://gcn.gsfc.nasa.gov/gcn/gcn3/2483.gcn3)

Soderberg, A. M., et al. 2004, ApJ, in press (astro-ph/0311050)

Stanek, K. Z., et al. 2003, ApJ, 591, L17

Tedds, J. A., \& Watson, M. G. 2004, in New Visions of the X-Ray Universe in the XMM-Newton and Chandra Era, ed. F. Jansen (ESA SP-488; Noordwijk: ESA), in press

Tedds, J. A., et al. 2003, GCN Circ. 2490 (http://gcn.gsfc.nasa.gov/gen/gcn3/ 2490.gcn3)

Tiengo, A., Mereghetti, S., Ghisellini, G., Rossi, E., Ghirlanda, G., \& Schartel, N. $2003, A \& A, 409,983$

Vaughan, S., et al. 2004, ApJ, 603, L5

von Kienlin, A., Arend, N., Lichti, G. G., Strong, A., \& Connell, P. 2003, Proc. SPIE, 4851, 1336

Watson, D., Reeves, J. N., Hjorth, J., Jakobsson, P., \& Pedersen, K. 2003, ApJ, 595, L29

Watson, D., Reeves, J. N., Osborne, J., O’Brien, P. T., Pounds, K. A., Tedds, J. A., Santos-Lléo, M., \& Ehle, M. 2002a, A\&A, 393, L1

Watson, D., Reeves, J. N., Osborne, J. P., Tedds, J. A., O'Brien, P. T., Tomas, L., \& Ehle, M. 2002b, A\&A, 395, L41

Yamazaki, R., Ioka, K., \& Nakamura, T. 2004, ApJ, submitted (astro-ph/0401142) 